\title{
Tumour Banking: The Spanish Design
}

\author{
M.M. Morente ${ }^{a} \quad$ E. de Alava ${ }^{b}$ P.L. Fernandez ${ }^{c}$ \\ aTumour Bank Unit, Molecular Pathology Programme, Spanish National Cancer Centre, Madrid; \\ bTumour Bank Unit, Molecular Pathology Programme, Cancer Research Centre-IBMCC, University of Salamanca-CSIC, \\ Salamanca, and 'Tumour Bank Unit, Department of Pathology, Hospital Clinic and IDIBAPS, Barcelona, Spain
}

\section{Key Words}

Biobank · Biorepository · Tumour bank · Tumour bank network $\cdot$ Translational research

\begin{abstract}
In the last decade the technical advances in high throughput techniques to analyze DNA, RNA and proteins have had a potential major impact on prevention, diagnosis, prognosis and treatment of many human diseases. Key pieces in this process, mainly thinking about the future, are tumour banks and tumour bank networks. To face these challenges, diverse suitable models and designs can be developed. The current article presents the development of a nationwide design of tumour banks in Spain based on a network of networks, specially focusing on its harmonization efforts mainly regarding technical procedures, ethical requirements, unified quality control policy and unique sample identification. We also describe our most important goals for the next years. This model does not correspond to a central tumour bank, but to a cooperative and coordinated network of national and regional networks. Independently from the network in which it is included, sample collections reside in their original institution, where it can be used for further clinical diagnosis, teaching and research activities of each independent hospital. The herein described 'network of networks' functional model could be useful for other countries and/or international tumour bank activities.
\end{abstract}

Copyright $\odot 2007$ S. Karger AG, Basel

M.M.M., E.d.A. and P.L.F. participated equally in the design and elaboration of the manuscript.

\section{KARGER \\ Fax +41613061234 \\ E-Mail karger@karger.ch}

www.karger.com
(C) 2007 S. Karger AG, Basel

$1015-2008 / 07 / 0744-0245 \$ 23.50 / 0$

Accessible online at:

www.karger.com/pat

\section{From Private/Institutional Collections to True Tumour Banks}

In Spain, as in most developed countries, a large number of private or public institutions keep biorepositories of human tumour samples. In fact, most of the currently accepted oncological entities were described in the 20th century by a clinical-pathological approach based on the study of tumours collected through collaborations between clinicians and pathologists. In parallel, and almost without effective connections with the clinical setting, basic researchers have produced a plethora of data, focusing on the main cancer basic mechanisms which mostly use cell lines and animal models.

The last decade of the 20th century was the scenario of a biotechnological revolution, characterised not only by new methods for genomic and proteomic studies but also by being able to effectively and efficiently transfer this technology to many research centres in the developed world as well as to its clinical setting. These technical developments have renewed the need for high-quality tissue samples, especially in an era when this technology is of increasing clinical value to [1-3]:

- Identify new parameters of clinical value, therefore demanding large-scale molecular studies with large numbers of cases.

- Transfer knowledge from basic to clinical research, which requires homogeneous tissue-sampling protocols for multi-centre studies.

- Confirm results obtained in the animal models and human cell lines, which requires providing human samples of clinical origin to basic research groups. 
- Predict treatment response, based on clinical trials including the acquisition of suitable clinical samples for molecular studies.

To face these challenges, diverse suitable models and designs can be developed: isolated banks or cooperative networks, prospective and/or retrospective collections, project or non-project driven, monographic or generic, private or public, commercial or non-profit, local vs. regional vs. national vs. international networks, etc. All these models would be useful or not, depending on their biorepository scope. Specific goals require specific designs, and the best practices for tumour banking depend on the repository goals.

\section{Tumour Bank Networking: The Spanish Design}

Tumour collections exist ever since pathology departments store blocks of tissue samples [4], but, due to the above-commented scientific goals, the current tumour bank definition was changed in order to bring a better service in translational research including harmonization within networking associated biorepositories and sharing samples between both applied and basic researchers [1-3].

Based on the original design of some national and regionally based initiatives, a peculiar tumour bank networking activity arose in the year 2000 in Spain. These activities share a common view about what tumour banking is supposed to be in the 21st century, including:

- Not only a tissue storage service, but also a series of hospital protocols that allow molecular studies of tumour samples.

- Collections, freezing and storage of neoplastic and normal tissues must be considered a routine in the departments of pathology and haematology.

- Not only a pathologists' activity but a global hospital activity involving surgeons, oncologists, haematologists, hospital managers and, obviously, pathologists.

- Homogeneous and suitable protocols for collection, handling, storage and use of frozen samples for research, teaching and cancer patient care.

- Being open to sharing tissue samples with basic and applied researchers.

- More specialized dedication of the personnel directly working in these units.

- Strict ethical protocols in order to guarantee the rights of the patient and society.

- Quality assurance and quality controls.

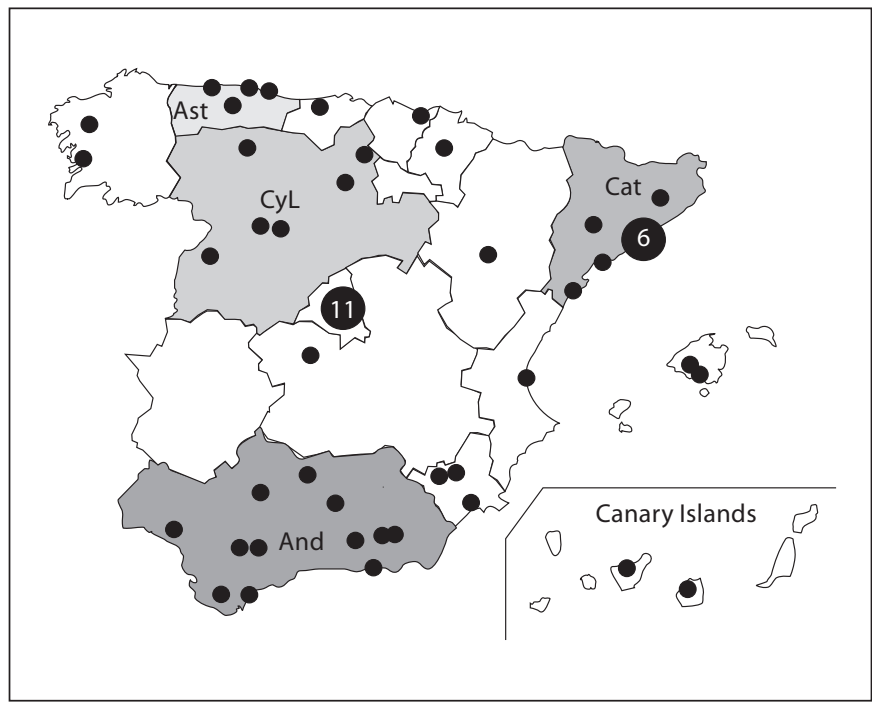

Fig. 1. Geographic localisation of associated tumour banks. And = Andalucía $;$ Cat $=$ Catalonia $; \mathrm{CyL}=$ Castilla y León; Ast $=$ Asturias.

- Cooperative tumour bank networks to allow multicentre and international clinical trials and collaborative studies.

The earliest of those initiatives was initiated by the Spanish National Cancer Centre (CNIO) in September 2000. The so-called Spanish National Tumour Bank Network (SNTBN) was created and is coordinated by the CNIO's Molecular Pathology Program, and aims to satisfy the demand of human neoplastic tissue under ideal conditions for the development of large-scale studies of clinical significance in Spain, and for a small high-quality series for basic researchers [5].

As a national centre, the first goal of this initiative is to promote high-quality tumour banks within every Spanish hospital, as a public service. After that, these banks are invited to participate in a collaborative network.

Along with this design, new networks arose from 2001 to 2005 in some Spanish autonomous regions: Castilla y León, Catalonia, Asturias, and Andalusia, all of them based on overtly collaborative goals (fig. 1). This regional development must be understood in the Spanish healthcare design. From the political point of view, Spain is composed by 17 autonomous regions (autonomous communities) with specific responsibilities including healthcare, which mainly belongs to the public sector.

The existence of four regional tumour banking networks and one with national scope, far from being a prob- 
lem, is a great platform of national collaboration, due to a common basic design. Independently from what network they are included in, sample collections reside in their original institution, where they can then be used for clinical diagnosis, teaching and research activities in each independent hospital. This generally accepted model does not correspond to a central tumour bank, but to a cooperative and coordinated network of hospital banks, based on homogeneous and optimal tissue-treatment protocols, and interconnected by a computer-based network.

Each network has a central coordination office that guarantees confidentiality, stability, QA/QC policy, homogeneous protocols, stable recognition by research agencies and ethic review boards, and leadership, following the so-called 'honest broker' role. Nevertheless, this design is hospital-based.

\section{Spanish Tumour Bank Network of Networks}

From 2003 this collaboration became more effective through the Thematic Network of Cooperative Research of Cancer Centres (RTICCC) promoted by the Carlos III National Institute of Health (Spanish Ministry of Health), which includes a specific tumour banking programme (TBP) with the involvement of 23 hospital tumour banks, most of them associated to either regional or national tumour banking networks.

During 2003 and 2004, the early RTICCC period, the TBP created a suitable framework of networking and harmonization in order to use the same technical protocols, the same homogeneous ethical requirements, a unique sample identification system and a common quality control programme (see below). All of these issues were agreed upon by consensus.

The development of the TBP was based on the confluence of the regional and supra-regional initiatives mentioned above and on the organization and financial support from the RTICCC, which in turn was based on: (a) development of regional networks; (b) the uniformity in the organization model, methodologies and in ethic-legal fundaments, and (c) the development of a quality control/ quality assurance policy.

The first two initiatives were started along with the programme, while the third started during the third year of the programme in order to test the quality of some aspects of it.

A coordination role was assigned to the $\mathrm{CNIO}$, based in Madrid, and the two main centres in Salamanca (CIC) and Barcelona (Hospital Clinic-IDIBAPS). This decision was based on two main reasons: firstly because cancer research in Spain is polarised in big centres in Madrid, Barcelona and Salamanca, and secondly the great expertise in tumour bank networking of the CNIO.

Once started, the TBP of the RTICCC, every local tumour bank coordinator was invited to join in task groups for the elaboration of consensus document, as well as to start an informative and supporting campaign with the regional agencies responsible for public health. Moreover, this campaign meant an impulse to the creation of welldeveloped regional networks.

The achievement of similar organization structures in each region, coupled with standardised protocols for the handling, storage and sharing of samples was considered of major importance for the success of the TBP and, therefore, future stable supra-regional networking. Thus, a consensus document dealing with organization, methodology, data-basing and ethical-legal issues was elaborated with the involvement of all the local coordinators from the RTICCC tissue banks [6].

Briefly, an organization scheme was proposed that consisted of a net of regional networks, each with a coordination node in charge of managing requests to the regional network and a central regional database. Methodology requirements dealt with time of ischemia, selection-freezing-storage processes and shipment according to international guidelines $[7,8]$. Indications and requirements regarding the design of databases capable of complying with the current legislation and necessary interconnections with other regions, as well as a central national database, were also included in the consensus documents. Finally, the anonymization of samples, the controlled access to files, the third-party sharing and a non-profit policy, were addressed in the final part of that document.

As stated before, the two above aspects were addressed, but not completed, during the two first years of the TBP. The evaluation of the achievements of such work had to take place within the last semester of the third year.

The quality policy was conceived to involve all the aspects of the tumour banking including procurement, preservation, shipment, data management, ethical-legal aspects, etc. not only in the local tumour bank, but at the regional/national network as well. Each bank was in charge of defining its own quality requirements according to its capabilities. Yet, a minimum of quality requirements were established in the consensus documents: less than half an hour for ischemia, selection of samples made by a pathologist (or trainee), at least $-80^{\circ} \mathrm{C}$ for storage, freezer alarms and back-up, informed consent, reviews carried out by a ethical committee, etc. 


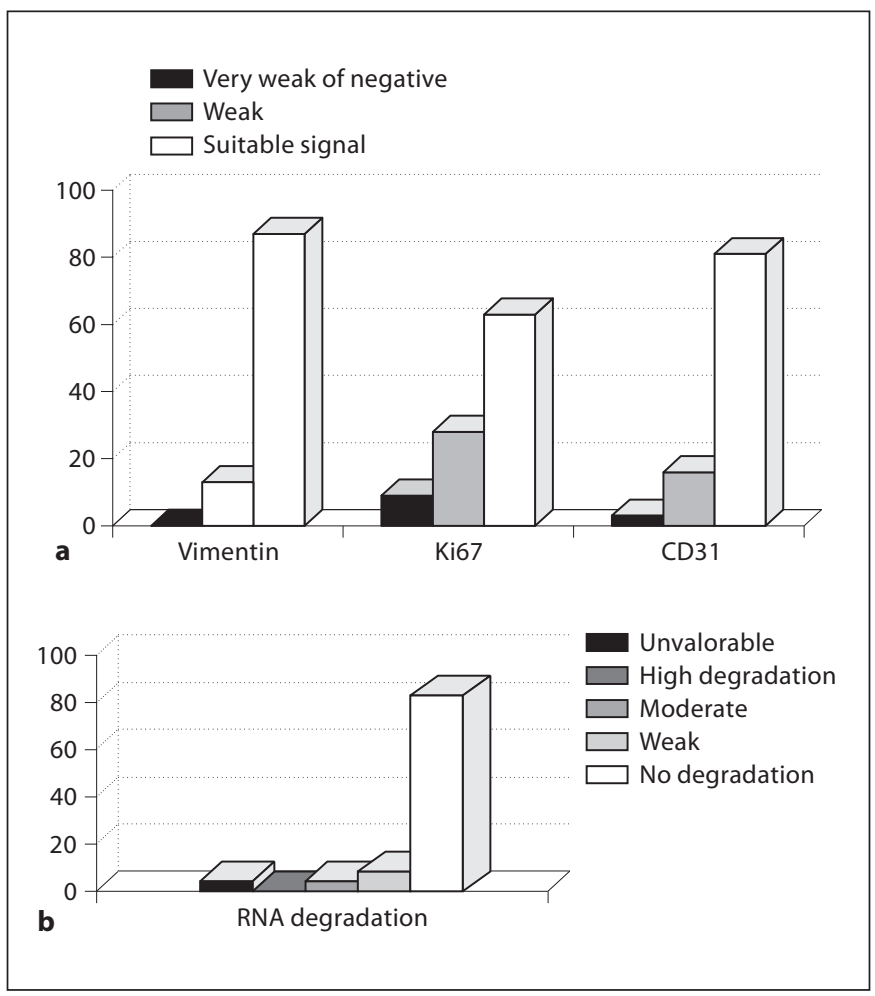

Fig. 2. First quality control results: antigenic preservation (a) and RNA integrity (b).

A control quality survey was designed and conducted in 2005 to test two randomly chosen samples of each bank for morphological, immunohistochemical (antigenic preservation of CD31, vimentin and MIB1 expression onto a tissue microarray with all the samples) and molecular quality (integrity of RNAm tested by Agilent Bioanalyz$\left.\mathrm{er}^{\circledR}\right)$. This first survey yielded very encouraging results given the good morphological, immunophenotype and molecular properties of the samples in most instances (fig. 2). Samples yielded good quality RNA in $97 \%$ of cases. The immunohistochemical results on paraffin-embedded tissue showed that samples had been processed and selected in an adequate manner, although there was a mild variability with respect to the MIB1 expression. In particular, there were a few samples (4\%) in which the expression of this epitope was either very weak or negative in tumour cells, suggesting the effect of a long fixation period of the samples used for the analysis. We then recommended to obtain specific paraffin blocks for each tumour bank whenever possible, with a very controlled fixation time, which are, in the experience of several members of the network, very useful for tissue microarray studies.

\section{General Procedures}

We here provide a brief summary of specific procedures and requirements which could be useful to those involved in tumour banking design and coordination.

\section{Collection of Specimens and Initial Evaluation}

The surgical specimens must be quickly transferred to the pathology department or frozen in situ after extraction in the operating theatre in order to minimize the action of hypoxic phenomena on genetic expression. The pathologist decides whether it is possible to separate one or more fragments for freezing without this operation affecting the correct pathological diagnosis of the process. Those areas with massive ischemia and/or necrotic phenomena must be avoided. For each frozen fragment, a fragment of similar dimensions should be selected in continuity with the frozen material for formalin fixation. This entire process must be carried out in the most aseptic conditions possible. Whenever possible, paired nonneoplastic tissue of the same organ is selected for freezing and for fixation and paraffin embedding.

\section{Freezing}

Samples must be frozen and duly identified in plastic cryomolds embedded in a cryosolidifiable medium through immersion in a highly cryogenic medium (i.e. isopentane below $-50^{\circ} \mathrm{C}$ ) that allows for rapid freezing. Freezing by direct immersion in liquid nitrogen is a valid method, although it is more damaging to the tissue and hinders subsequent microscopical examination, especially when microdissection techniques are required.

\section{Identification of Samples}

Each sample to be processed by the tissue bank is immediately identified in accordance with the tissue identification protocol, with clear reference to the tissue type (normal or pathological). This identification must be mechanized, whenever possible, for greater clarity of references. The final identification of the samples can be carried out using a double system of barcodes and alphanumeric characters for generating labels for identifying preparations, blocks, reports, etc.

\section{Conservation of Frozen Tissue and Security Systems}

The blocks of neoplastic and non-neoplastic tissue frozen and included in cryomolds with OCT compound must be properly identified so that they are not used for 
diagnosis, except when this is absolutely necessary. Tissues are stored at temperatures below $-75^{\circ} \mathrm{C}$ in chest freezers with the necessary security measures to avoid thawing and/or exposure to excessive changes in temperature. They include: inclusion in the emergency hospital's secure electricity supply, triple alarm system (local visual and acoustic, remote to hospital personnel, telephone to tumour bank members, etc.), $\mathrm{CO}_{2}$ injection system backup and spare nearby empty freezer.

\section{Ethical Aspects}

All neoplastic tissues accepted by the tumour bank originated from the healthcare activity of the associated hospitals, in particular biopsies, surgical specimens, and necropsy samples, absolutely respecting the procedures that guarantee correct ethical activity. These may be summarized in the following basic guidelines:

- In no case may the correct diagnosis of a sample be compromised by reason of the collection of material for the tissue bank.

- The central database shall not contain information that could allow the direct or indirect identification of patients, each associated hospital being responsible for maintaining the codes for such anonymity.

- The associated hospitals must present the activity protocols of the tumour bank network to their corresponding ethical and clinical research committees, so that they may be studied and approved.

- The associated hospitals are obliged to obtain a document-based informed consent that indicates the patient's approval that surplus tissue remaining following its use for the correct histopathological diagnosis may be used for research purposes.

- These same ethics and research committees of the various hospitals must be aware of and positively report the standard working methodology of the hospital tumour bank beforehand the signing of the collaborative agreements.

\section{Data Security}

Currently, different networks use their own specific computer application supporting the activity and collections of the tumour bank network. All of them with some basic principles:

1 Hospital databases may contain personal information that permit the identification of the patient (given and family names, clinical record number, pathology department reference, and member of staff in charge of the case). However, all these data will remain coded.

Tumour Banking: The Spanish Design
2 No personal information will be accessible to users outside the hospital (i.e. tumour bank network coordinators, researchers).

3 To allow the correct use of the database, each case will undergo a double disassociation procedure by which an identification code will be generated that will be the only one used in the central database. A similar step will be carried out when samples are distributed to researchers.

4 Information is passed through a private network over an RDSI-type connection or Internet secure pathway.

5 The central database will contain no fields that allow patients to be identified. The strictest precautions set out in the Spanish Organic Law of the Regulation of Automatic Treatment of Data will be taken (level 3).

6 All access to hospital and central databases will be conducted through authentication mechanisms involving a username and password, different levels of operational access being established to limit/block the possible modification of records.

\section{Future Goals and Perspectives}

Soon after the elaboration of this article, the TBP coordination team will finish evaluating the 2003-2006 periods, and the RTICCC will be evaluated for a second period (2007-2010). Once more, the TBP is going to be an essential part of this development. On the other hand, the Spanish Parliament is preparing an Act on biomedical research, which will deal with biobanks in general, and tumour banks in particular. The adjustment to the forthcoming new Spanish legal framework in biobanking will be a crucial objective for the following period. In preparing this Act, the National DNA Bank, through the Carlos III National Institute of Health (Spanish Ministry of Health) supporter of the RTICCC, conducted a survey to all biobanks in Spain at the end of 2005.

The results of this survey helped to evaluate the programme and to define future goals for the TBP. They showed that the TBP has achieved a high quality, and a good adequacy to the future legal framework, although it also identified several challenges, which will also be a priority to our TBP. Some of the aims for the following period will be:

a. To develop functional and safe databases, in which an adequate amount of clinical and pathological information could be linked to the samples. A consensus needs to be reached for the fields which need to be included in the databases (i.e. which minimum clinical and epide-

Pathobiology 2007;74:245-250 
miological information needs to be linked to breast carcinoma samples). These databases need to be registered in the National Data Protection Agency, and would correspond to the regional/interregional networks. Additionally, there should be a central, national database with a short but common number of fields, to enable the interregional exchange of samples and information. A common systematized nomenclature also needs to be adopted both for topographical and morphological terms.

b. Informed consent needs to be obtained to store or to use for research all new samples. Although more than $90 \%$ of the stored samples do have an informed consent, new procedures to obtain consent are needed, especially in order to give the patient the possibility of reversing his/her consent.

c. Our TBP usually stores solid pieces of tumour samples from patients with tumours. It will therefore be important for the TBP to also be able to store plasma, DNA, and (in some cases) cell suspensions, not only from tumour patients, but also from patients who have a genetic susceptibility for cancer (i.e. in familiar breast/colorectal/endocrine cancer).

d. The final responsibility of allowing or rejecting sample requests usually corresponds to the tumour bank director. External scientific and ethical committees need to be implicated in all the institutions to help TBP coordinators to evaluate and decide about the worthiness and adequacy of a given project to receive or not to receive samples.

e. The conformity of stored samples and tumour bank services to standards provides assurance about their quality, safety and reliability. Only one third of tumour banks in Spain have got a quality certificate (i.e. ISO 9001: 2000 ), and about another $25 \%$ are in the process of obtaining it. Our goal is to obtain UNE-EU-ISO 9001:2000 certifications at least for the central laboratories of the regional networks in 2007.

f. We have had strong support from the regional and national administration. To gain support from the general population, a firm divulgation programme needs to be set up so that citizens can understand and back our activities, first of all by giving their informed consent. Continued effort, however, will be necessary in order to increase the support from public agencies to receive more financial, human and technical resources.

In conclusion, we would like to stress that tumour banks are not a goal in themselves, but a tool to support translational research in cancer. Therefore, a good parameter to evaluate their activity is not only the number of samples stored but rather the number of cooperative projects/publications of excellence in which a tumour bank programme is involved. The current achievement of TBP in Spain will only continue to work with strong ethical and quality standards, and will also depend on the use of TBP samples for projects of excellence. Our 'network of networks' functional model could be useful for other countries and/or international tumour bank activities $[9,10]$.

\section{Acknowledgements}

Spanish Tumour Bank Network of Networks is funded in part with public funds from the Instituto de Salud Carlos III (Spanish Ministry of Health), under Cancer Centres Thematic Network ISCIII-RETIC RD06/0020.

\section{References}

1 Fernández PL, Ferrer B, Campo E: Bancos de tumores: papel en la investigación biomédica del presente y el futuro. Cir Esp 2004;76: 4-8.

$>2$ Morente MM, Alonso S: Current challenges of human tumour banking. Hematol Oncol 2005;23:54-56

3 Oosterhuis JW, Coebergh JW, van Veen EB: Tumour banks: well-guarded treasures in the interest of patients. Nat Rev Cancer 2003; 3:73-77.

4 Grizzle WE, Woodruff KH, Trainer TD: The pathologist's role in the use of human tissues in research - legal, ethical, and other issues. Arch Pathol Lab Med 1996;120:909-912.

5 CNIO Tumour Bank Network website: http://www.cnio.es/ing/programas/progTumor01.asp
6 RTICCC Tumour Bank Program website: http://www.rticcc.org/articulos/p2/protocolos-p2/index.shtml

$>7$ International Society for Biological and Environmental Repositories (ISBER): Best practices for repositories. I. Collection, storage, and retrieval of human biological materials for research. Cell Preserv Technol 2005; 3:5-48.

8 Morente MM, Mager R, Alonso S, Pezzella F, Spatz A, Knox K, Kerr D, Dinjens WNM, Oosterhuis JW, Lame KH, Oomen MHA, van Damme B, van de Vijver M, van Boven $\mathrm{H}$, Kerjaschki D, Pammer J, Lopez-Guerrero JA, Llombart-Bosch A, Carbone A, Gloghini A, Teodorovick I, Isabelle M, Passioukov A, Lejeunek S, Therassek P, van Veenl EB, Ratcliffe C, Riegman PHJ: TuBaFrost 2: standardising tissue collection and quality con- trol procedures for a European virtual frozen tissue bank network. Eur J Cancer 2006;42: 2684-2691.

-9 Knox K, Kerr DJ: Establishing a national tissue bank for surgically harvested cancer tissue. Br J Surg 2004;91:134-136.

10 Riegman PHJ, Dinjens WNM, Oomen MHA, Spatz A, Ratcliffe C, Knox K, Mager R, Kerr D, Pezzella F, van Damme B, van de Vijver $\mathrm{M}$, van Boven $\mathrm{H}$, Morente MM, Alonso S, Kerjaschki D, Pammer J, Lopez-Guerrero JA, Llombart-Bosch A, Carbone A, Gloghini A, Teodorovick I, Isabelle M, Jamine D, Passioukov A, Lejeune S, Therassek P, van Veenl EB, Lame KH, Oosterhuis JW: TuBaFrost 1: uniting local frozen tumour banks into a European network - an overview. Eur J Cancer 2006;42:2678-2683. 